\title{
Rapidly evolving circularly polarized emission during the 1994 outburst of GRO J1655-40
}

\author{
J.-P. Macquart ${ }^{1}$, K. Wu ${ }^{2}$, R. J. Sault ${ }^{3}$, and D. C. Hannikainen ${ }^{4,5}$ \\ 1 Kapteyn Astronomical Institute, University of Groningen, Postbus 800, 9700 AV Groningen, The Netherlands \\ 2 Mullard Space Science Laboratory, University College London, Holmbury St Mary, Surrey RH5 6NT, UK, \\ and School of Physics A28, University of Sydney, NSW 2006, Australia \\ e-mail: kw@mssl.ucl.ac.uk \\ 3 Australia Telescope National Facility, Narrabri, NSW 2390, Australia \\ e-mail: rsault@atnf.csiro.au \\ ${ }^{4}$ Department of Physics and Astronomy, Southampton University, Southampton, SO17 1BJ, UK \\ 5 Observatory, PO Box 14, 00014 University of Helsinki, Finland \\ e-mail: diana@astro.helsinki.fi
}

Received 11 April 2002 / Accepted 24 September 2002

\begin{abstract}
We report the detection of circular polarization during the 1994 outburst of the Galactic microquasar GRO J1655-40. The circular polarization is clearly detected at 1.4 and $2.4 \mathrm{GHz}$, but not at 4.8 and $8.4 \mathrm{GHz}$, where its magnitude never exceeds $5 \mathrm{mJy}$. Both the sign and magnitude of the circular polarization evolve during the outburst. The time dependence and magnitude of the polarized emission can be qualitatively explained by a model based on synchrotron emission from the outbursts, but is most consistent with circular polarization arising from propagation effects through the relativistic plasma surrounding the object.
\end{abstract}

Key words. black hole physics - galaxies: active - galaxies: jets - polarization - radio continuum: galaxies X-rays: individual: GRO J1655-40

\section{Introduction}

GRO J1655-40 (Nov Sco 1994) is a transient X-ray binary at an estimated distance of $3.2 \mathrm{kpc}$ (Hjellming \& Rupen 1995) containing a low mass F3IV-F6IV companion star and a blackhole candidate which has a mass about $7 M_{\odot}$ (Orosz \& Bailyn 1997; Soria et al. 1998; Shahbaz et al. 1999). It was first detected in the hard X-ray band by the Burst and Transient Source Experiment (BATSE) on board the Compton GammaRay Observatory on 1994 July 27, at the onset of an X-ray outburst (Zhang et al. 1994). Its X-ray flux in the 20-100 keV band quickly reached 1.1 Crab on August 1. The system remained in an outburst state until about August 15 and then was briefly quiescent, but flared again on September 6 (Harmon et al. 1995). GRO J1655-40 underwent several further outbursts between 1994 and 1997 before retreating into a quiescent state in late 1997.

The radio counterpart of GRO J1655-40 was first identified by the Molongo Observatory Synthesis Telescope (MOST) at $843 \mathrm{MHz}$ (Campbell-Wilson \& Hunstead 1994a) shortly after the BATSE discovery. The radio flux density showed a steep initial rise, and it reached 4.2 and 5.5 Jy on 1994 August 14

Send offprint requests to: J.-P. Macquart, e-mail: jpm@astro.rug.nl and 15 respectively (Campbell-Wilson \& Hunstead 1994b). Imaging using the Very Large Array (VLA), the Very Long Baseline Array (VLBA), and the Southern Hemisphere VLBI Experiment (SHEVE) showed repeated episodes of relativistic ejections from the system (Hjellming \& Rupen 1995; Tingay et al. 1995), with the ejecta in superluminal motion. After correction for the inclination to the line of sight, the ejection velocity was inferred to be $v \sim 0.92 c$ in the rest frame of the system (Hjellming \& Rupen 1995).

During the 1994 ejection events, GRO J1655-40 was monitored by the MOST, the Australian Telescope Compact Array (ATCA) and the Hartebeesthoek Radio Astronomy Observatory (HartRAO) (see Hannikainen et al. 2000 for details). The radio emission showed strong (up to about 10\%) linear polarization. The variations in the multi-frequency linear polarization light curves reveal several ejection events and strong opacity effects. The simultaneous peaking of multifrequency radio light curves at about August 19 contradicts the prediction of the conventional synchrotron bubble models (e.g. Hjellming \& Johnston 1988). This behaviour is, however, consistent with that of the generalized-shock model for AGN jets (Marscher \& Gear 1985), for the shocks in the jets of GRO J1655-40 in the initial growth stage (Wu et al. 2002; Stevens et al. 2002). 
Here we report the detection of circular polarization in the radio emission from GRO J1655-40. Circular polarization has been detected in two other Galactic jet sources: SS433 (Fender et al. 2000) and GRS 1915+105 (Fender et al. 2002). For SS433 the observations contained data from two epochs only; for GRS $1915+105$ the observations were conducted only after (as opposed to during) the onset of the ejection episodes and their associated radio emission.

Circular polarization is also observed in a broad variety of AGN, from relatively weak sources like Sgr A* and M 81* (Bower et al. 1999; Sault \& Macquart 1999; Brunthaler et al. 2001) to powerful quasars such as PKS 1519-273, 3C 273 and 3C 279 (Wardle et al. 1998; Homan \& Wardle 1999; Macquart et al. 2000). The origin of the circular polarization is uncertain (Wardle \& Homan 2001; Macquart 2002).

The two most likely causes of the circular polarization in Galactic jet sources are the small amount intrinsic to synchrotron radiation, and that which results from the propagation of linearly polarized radiation through a relativistic plasma (Legg \& Westfold 1968; Pacholczyk 1973; Cheng et al. 1985; Fender et al. 2002). Identification of its cause and relationship to other observable jet parameters can constrain, for instance, the properties of the magnetic field in the jet and the low energy end of the relativistic electron distribution. This in turn may indicate whether the composition of the relativistic jets is primarily leptonic or ionic ( $\mathrm{e}^{-} / \mathrm{p}$ ) (Wardle et al. 1998; but see also Ruszkowski \& Begelman 2002).

In determining the origin of the circular polarization, it is pertinent to compare the properties of circular polarization observed in AGN with that in Galactic jet sources. The magnitude of the circular polarization is similar in both classes of source. Despite this, it has not been possible to identify the origin of the circular polarization in either SS 433 or GRS 1915+105.

Here we report variable circularly polarized emission during the 1994 August-September outbursts of GRO J1655-40. The spectral and temporal coverage of the circular polarization over the duration of the flaring in this source presents an exceptional opportunity to quantify the properties of the circular polarization in a Galactic X-ray binary. In particular, this is the first time that the circular polarization has been measured during the commencement of the radio flare, during which the source appears to exhibit dramatic polarization variability.

We discuss the observations in Sect. 2 and present a modelindependent interpretation of the data in Sect. 3. These results are discussed in the context of a model for the outbursts in GRO J1655-40 in Sect. 4, and the characteristics of the circular polarization in this source are related to those of other X-ray binaries and AGN. The conclusions are presented in Sect. 5.

\section{Observations and results}

\subsection{Data reduction}

The data presented in this paper were obtained with ATCA over the interval 1994 August 15 to 1994 September 3 (TJD $=9579$ to 9597). The ATCA is an Earth-rotation aperture synthesis array, comprising six $22 \mathrm{~m}$ antennas which can be moved along an east-west track to give baselines up to $6 \mathrm{~km}$
(Frater et al. 1992). The observations of GRO J1655-40 were made at centre frequencies of 1.380, 2.378, 4.800, 5.904, 8.640 and $9.200 \mathrm{GHz}$ with $128 \mathrm{-MHz}$ bandwidth in two orthogonal polarizations. Each observation was typically $10 \mathrm{~min}$ in duration, except on August 15, where the observations spanned 3 hours at 1.380 and $2.378 \mathrm{GHz}$ and 5 hours at 4.800 and $8.640 \mathrm{GHz}$. The antenna gain and phase calibration were derived from regular observations of the point source calibrator PKS 1740-517. The flux density scale was tied to the ATCA primary flux calibrator PKS 1934-638 (Reynolds 1994). The array was in the $6.0 \mathrm{~A}$ configuration throughout these observations, giving interferometer spacings from 337 to $5939 \mathrm{~m}$. The data from the bands centred at 5.904 and $9.200 \mathrm{GHz}$ have been excluded from the present discussion, since the stability of the instrumental response to circular polarization is not well characterized at these frequencies.

Data reduction was performed in the MIRIAD package (Sault et al. 1995). The instrumental leakages and antenna gains were determined simultaneously using either PKS 1934-638 or PKS 1740-517. At all wavelengths a single round of phase self-calibration was performed on the GRO J1655-40 data (using a point source model) to eliminate residual phase instability.

The reduction of this dataset for variations in the total and linearly polarized flux density is discussed further in Hannikainen et al. (2000). However, some specific comments relating to the circular polarization are in order.

Normal polarization calibration of ATCA data assumes the "weakly polarized" case (Sault et al. 1991), where the terms in "leakage $\times(Q, U, V)$ " are neglected. The "weakly polarized" case does not calibrate leakage between linearly and circularly polarized emission. The MIRIAD calibration routines allow solution using the "strongly polarized" case, which also solves for second order terms. To determine such a calibration solution requires a calibrator source which is at least a few per cent linearly polarized, so that there is sufficient signal in the second order terms.

In the present case, the lack of observation of an appropriate linearly polarized source forced the use of the weakly polarized solutions to determine the instrumental leakage. This presents a limitation to the polarimetric accuracy of the circular polarimetry; the ATCA can measure circular polarizations to a level $V / I<0.01 \%$ (Rayner et al. 2000) provided the leakages are determined using the strongly polarized solutions. However, extensive comparison against the strongly-polarized solutions indicates that use of the weakly polarized equations results in an uncertainty in Stokes $V$ at worst $5 \%$ of the total linearly polarized flux density (Rayner 2000). The level of the circular polarization detected in GRO J1655-40 is sufficiently high relative to the linearly polarized flux density (hereafter denoted $\mathcal{P}$ ) that we are confident of the reality of the circular polarization in this source. We further note that polarimetric leakages are quite stable over time scales of several days on the ATCA.

We have adopted a limit of $0.05 \mathcal{P}$ due to polarization leakage as the total absolute error in quoting errors for the circular polarization. However, several arguments suggest this limit is conservative. 

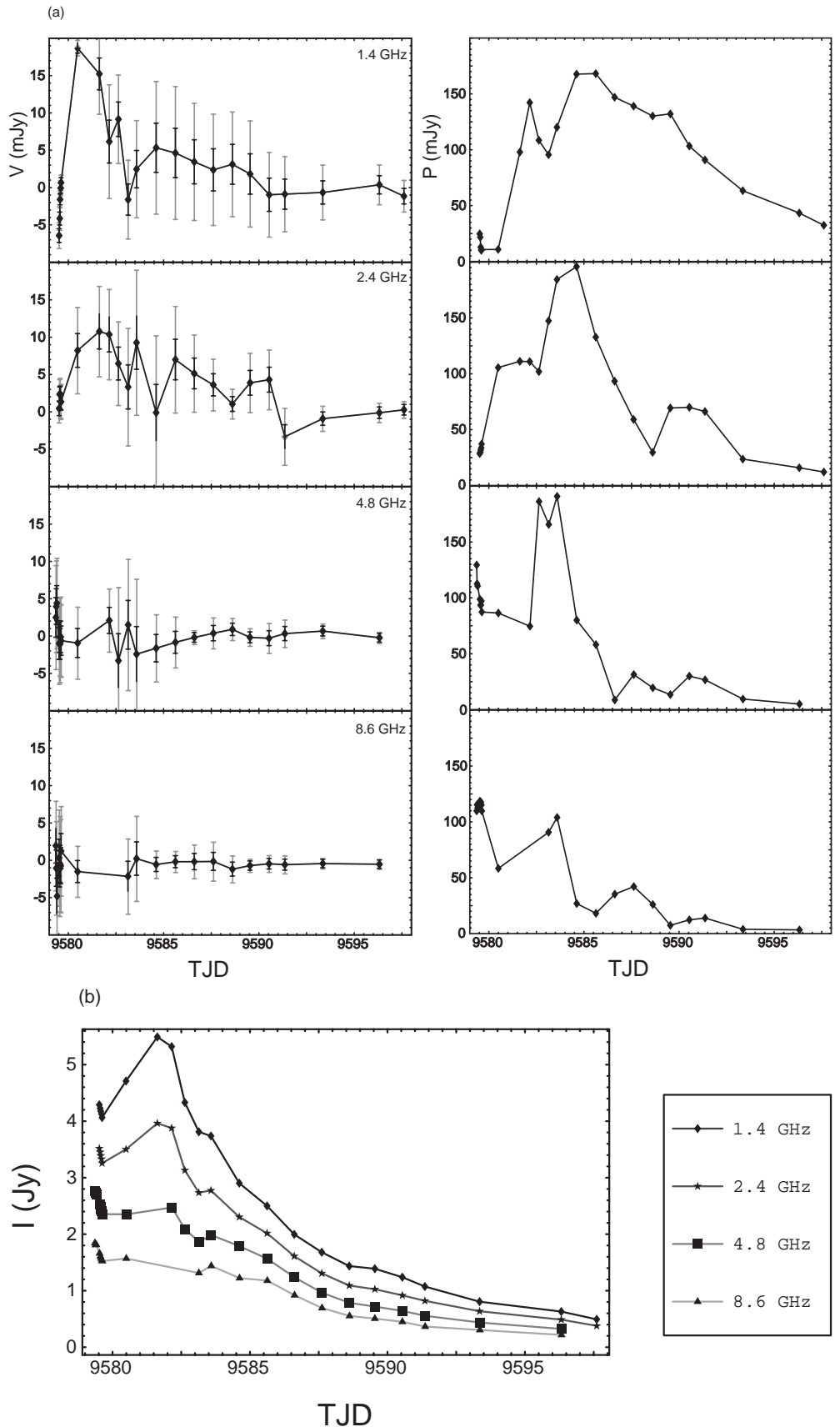

Fig. 1. a) Evolution of the circular (left) and linear (right) polarization in GRO J1655-40 at 1.4, 2.4, 4.8 \& 8.6 GHz (TJD = JD-2 440 000.5). The black error bars for the circular polarization values represent the combination of $1 \sigma$ thermal noise errors with the one third of the maximum possible error due to antenna leakage uncertainties; as such the black error bars should be interpreted $1 \sigma$ error bars in both random and systematic error. The gray error bars represent the 1- $\sigma$ thermal noise errors combined with the maximum possible uncertainty due antenna leakage, which is $0.05 \times \mathcal{P}$ (see text). The circular polarization variations at TJD 9579 at $1.4 \mathrm{GHz}$ are reproduced in greater detail in Fig. 2 . The linear polarization plots are adopted from Hannikainen et al. (2000), and the errors in these curves are $\sim 1 \mathrm{mJy}$. b) Lightcurves of the variations in Stokes $I$ at the same four frequencies.

The linear polarization does not follow the same trends as the circular polarization. In order to explain a substantial fraction of the variations in Stokes $V$ as an effect of instrumental leakage, this would require large variations in the degree of polarization leakage on the time scale of the observed variability, i.e. from day to day. This is at variance with the fact that leakages are known to be stable on ATCA over periods exceeding several days.
Some specific limits are provided by detailed examination of the data.

- 1.4 GHz: the circular polarization measured between the two days TJD 9581 and 9582 are of comparable magnitude, even though the linear polarization increased sharply between these two epochs. The linearly polarized flux density is $<10 \mathrm{mJy}$ on TJD 9581, while the circularly polarized 
flux density is $18 \mathrm{mJy}$ with a $0.5 \mathrm{mJy}$ error due to thermal noise. However, when the linear polarization increases to 150 mJy on TJD 9582, the circular polarization is measured at $15 \mathrm{mJy}$. Thus, if the variation in $V$ over this time interval is entirely due to instrumental leakage, the small change in $V$ compared with the large change in linear polarization implies that instrumental leakage contaminates the circular polarization at a level less than $0.02 \mathcal{P}$.

Moreover, we note that the general increase in linear polarization in the interval TJD 9581-9586 is not mirrored by an increase in the circular polarization;

- At $2.4 \mathrm{GHz}$ the linear polarization reaches $194 \mathrm{mJy}$ on TJD 9584 but $V$ does not greatly exceed $10 \mathrm{mJy}$ over the entire period of observation. The leakage is thus smaller than $5.5 \%$ of the linearly polarized flux density;

- At $4.8 \mathrm{GHz}$ the linear polarization reaches $191 \mathrm{mJy}$ but at no stage during or after the flaring does $V$ exceed $5 \mathrm{mJy}$. This suggests instrumental calibration results in a leakage of less than $2.3 \%$ of the linear polarization into circular polarization.

\subsection{Results}

Figure 1 shows the circular polarization and linear polarization from 1.4 to $8.6 \mathrm{GHz}$ during the flaring.

The circular polarization lightcurves at 1.4 and $2.4 \mathrm{GHz}$ are characterised by rapid changes over the first three days (TJD 9579-9582). At $1.4 \mathrm{GHz}$ the circular polarization changes from $-6.4 \pm 1.7 \mathrm{mJy}$ to $+0.6 \pm 1.0 \mathrm{mJy}$ over a period of $150 \mathrm{~min}$ at the start of the outburst, on TJD 9579 (see Fig. 2). The sign change is confirmed on the following day, when the circular polarization is measured at $18.0 \pm 1.0 \mathrm{mJy}$. Less pronounced variability is observed at $2.4 \mathrm{GHz}$ over the corresponding interval, and there is no evidence for a sign change during the initial outburst at this frequency. At both frequencies the circular polarization then rapidly decreases from this maximum value, but shows a relatively sustained level of $\approx 4 \mathrm{mJy}$ from TJD 9584-9588.

Several marginally significant dips are apparent in the circular polarization lightcurves. There is a marginally significant dip at $2.4 \mathrm{GHz}$ on TJD 9591.36, where the circular polarization is negative before it returns to zero. The detections of other "dips" in the circular polarization lightcurves, at $1.4 \mathrm{GHz}$ at TJD 9582.15 and 9583.14 and $2.4 \mathrm{GHz}$ at TDJ 9583.14 and 9584.61, are not significant if the telescope leakage is as high as $5 \%$, and are marginally significant if the leakage is closer to $2 \%$ (see Sect. 2.1).

There is no clear detection of circular polarization at 4.8 or 8.6 GHz. The circular polarization never exceeds $5 \mathrm{mJy}$ at either frequency. It is only possible to set restrictive upper limits on the circular polarization at late epochs when the degree of linear polarization is low. After TJD 9589 the circular polarization is consistent with zero, with measured values between $0.6 \pm 1.0 \mathrm{mJy}$ and $-0.3 \pm 2.0 \mathrm{mJy}$ at $4.8 \mathrm{GHz}$ and between $-0.7 \pm 1.5$ and $-0.5 \pm 1.5 \mathrm{mJy}$ at $8.6 \mathrm{GHz}$.

Figure 1 shows that several large changes in the 1.4 and $2.4 \mathrm{GHz}$ circular polarization lightcurves bear a close resemblance to the flares identified in the linear polarization by Hannikainen et al. (2000), as marked. For instance, the rise and decay of the first circularly polarized outburst shows a corresponding burst in the linear polarization over the same epoch, from TJD 9580-9583.

While some features in the circular polarization lightcurves at 1.4 and $2.4 \mathrm{GHz}$ appear to be connected to some in the linear polarization, the shapes of the lightcurves are qualitatively different. This suggests that the fine structure of the lightcurves observed in $V$ is real, and not due to instrumental leakage from linear to circular polarization.

\section{Analysis}

Only a small fraction of the source emission appears to be circularly polarized. The circular polarization lightcurve-like the linear polarization lightcurve - shows features which are not reflected in the total intensity. Many of the polarized "outbursts" therefore contribute little to the total source emission.

One can estimate the degree of polarization at $1.4 \mathrm{GHz}$ on TJD 9579 if the rapid changes in $V$ are connected with those in $I$. By comparing the rapid changes in the polarized and total intensity over this interval, the circular and linear polarizations of the variable component are estimated to be $-3.3 \pm 0.4 \%$ and $9.8 \pm 1.5 \%$ respectively at this frequency (see Fig. 3). We cannot estimate the degree of polarization at higher frequencies using this method because the changes in $V$ are not clearly detected. Fender et al. (2000) speculated that, although $\bar{V} / \bar{I} \sim 0.3 \%$ is measured in SS 443 , the actual circular polarization of the polarized component was likely be an order of magnitude higher. Our measurement substantiates this speculation for GRO J1655-40.

It is impossible to explain the behaviour of the circular polarization in terms of the bursting and decay of a single righthanded circularly polarized component in the source. We suggest that the evolution of the circular polarization is associated with the evolution of the multiple bursts emanating from the source, each of which is circularly polarized. There is both transient right-handed and left-handed circularly polarized emission at various times. The negative circular polarization clearly detected at early epochs cannot reflect the quiescent level of the circular polarization since its level at later epochs, after the bursts, tends towards zero. Furthermore, the dip in the circular polarization at $2.4 \mathrm{GHz}$ (TJD 9591.36) also appears to be negative, suggesting that some component of the source "bursts" in negative circular polarization.

We argue that the rapid decline of the (positive) circular polarization at around TJD 9581-9583 is due to increased generation of negative circular polarization from another subcomponent in the source, rather than decay of the component responsible for the positively polarized emission. The fact that the initial flare decays extremely rapidly at 1.4 and $2.4 \mathrm{GHz}$ supports this. Although the data is clearly marginal, the circular polarization also appears to rebound to higher (positive) values and decay more slowly after TJD 9584.5. 


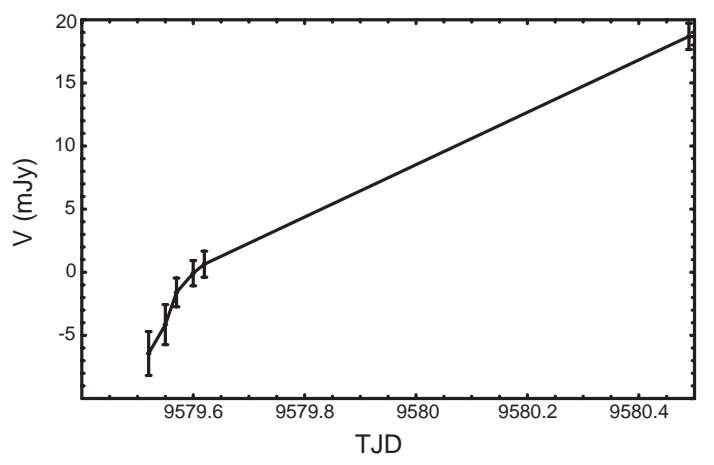

Fig. 2. A blow-up of the lightcurve of Stokes $V$ at $1.4 \mathrm{GHz}$ during the onset of the radio outburst. The errors in the values plotted are dominated by thermal noise, not antenna leakage uncertainties.

(a)

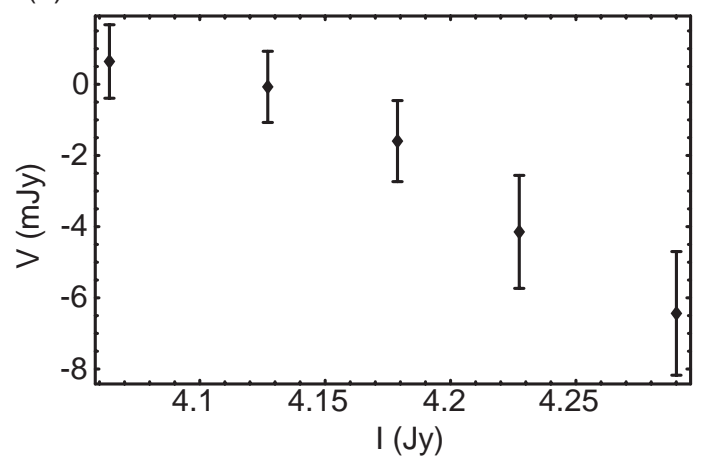

(b)

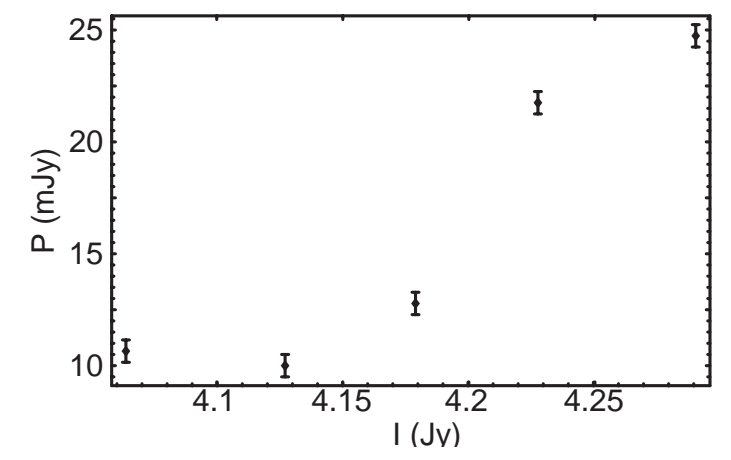

Fig. 3. a) The relationship between $V$ and $I$ on TJD 9579 at $1.4 \mathrm{GHz}$. If changes in the two quantities with time are related, this implies the presence of a polarized component of the source with $-3.3 \pm 0.4 \%$ circular polarization at $1.4 \mathrm{GHz}$. b) The corresponding relationship between $\mathcal{P}$ and $I$ at $1.4 \mathrm{GHz}$. A fit to the earliest four epochs (the rightmost four points) suggests a linear polarization of $9.8 \pm 1.5 \%$ in the varying component. The point with $I=4.064 \mathrm{Jy}$ corresponds to the last epoch on the first day of observations, at TJD 9579.62.

\section{Discussion}

\subsection{Origin of circular polarization}

The circular polarization is most plausibly either intrinsic to synchrotron emission or arises as a result of polarization conversion through relativistic plasma associated with the source (see Pacholczyk 1977). Other causes of circular polarization are less likely. The angular diameter of the GRO J1655-40 is too large for scintillation-based circular polarization
(Macquart \& Melrose 2000) to be relevant here. Emission from coherent emission processes is also unlikely, since the total flux density and linearly polarized emission are already well explained by synchrotron models (Hannikainen et al. 2000; Wu et al. 2002).

The circular polarization is negative at the onset of the radio flare on TJD 9579, but changes sign and peaks on TDJ 9580. The spectrum is inverted at the low frequencies when the sign change of the circular polarization occurs at TJD 5979 and subsequently evolves to a pure power law after $\sim 1$ day (Hannikainen et al. 2000).

In principle, both synchrotron radiation and polarization conversion are capable of explaining both the magnitude and sign changes observed in the circular polarization. Both predict a change in the sign as the emission evolves from optically thick to thin (Melrose 1971; Pacholczyk 1977).

The degree of circular polarization expected from synchrotron radiation from particles with Lorentz factor $\gamma$ in a homogeneous magnetic field is $m_{\mathrm{C}} \sim 1 / \gamma$. If the synchrotron optical depth, $\tau$, exceeds unity at early epochs, as suggested by the data, the $-3.3 \%$ circular polarization deduced from the $V$ and $I$ variations on TJD 9579 could be due to the synchrotron emission from electrons with $\gamma \lesssim 30$. The low degree of linear polarization, $m_{\mathrm{P}} \approx 9.8 \pm 1.5 \%$, deduced from the corresponding $P$ and $I$ variations is consistent with the value expected from optically thick synchrotron emission. For particles with energy distribution $N(\gamma) \mathrm{d} \gamma \propto \gamma^{-s} \mathrm{~d} \gamma$, one expects $m_{\mathrm{P}}=3 /(6 s+13)$ (e.g. Pacholczyk 1977). The linear polarization then implies $s \approx 2.9 \pm 0.9$. This value is consistent with the spectral index of -0.7 estimated in Wu et al. (2002) and Stevens et al. (2002). Lower values of $\gamma$ and $s$ are required if the magnetic field in the emission region is inhomogeneous and causes depolarization.

On the other hand, the emission may originate in an optically thin sub-component of the source. One then needs to account for the discrepancy between the observed degree of linear polarization and the canonical value $m_{\mathrm{P}_{\tau<1}}=(s+1) /(s+7 / 3) \sim$ $70 \%$ expected for optically thin synchrotron radiation.

The most likely explanation is that the observed emission is composed of contributions from several sub-regions, each with different intrinsic polarizations, caused by variations in the orientation of the magnetic field. In this case the circular and linear polarizations would both be depolarized. Thus the intrinsic circular polarization is higher than the measured value. The intrinsic circular polarization would be of order $\sim 3.3 \times(70 \% / 10 \%)=23 \%$ if the depolarization affects both linear and circular polarization to a comparable degree. This would require the circular polarization to originate from electrons with $\gamma \lesssim 4$.

An external Faraday screen can also account for the (linear) depolarization, but this is unlikely since the measured Faraday rotation in the source is only moderate: $\sim 20-100 \mathrm{rad} / \mathrm{m}^{2}$ (Hannikainen et al. 2000).

The circular polarization could result from the propagation of linearly polarized radiation through a relativistic plasma. This circular polarization arises because the wave modes in a relativistic plasma are elliptical, and propagation through the plasma converts linear polarization to circular polarization, 
similar to the way in which Faraday rotation converts radiation between Stokes $Q$ and $U$ in a cold plasma.

This mechanism is easily capable of yielding circular polarizations of a few percent (Ruszkowski \& Begelman 2002). Circular polarization produced via this effect is strongest for optical depths near unity. As such, our data qualitatively favours this model, because the peak of the circular polarization at TJD 9580 coincides with the date at which $\tau \sim 1$ at $1.4 \mathrm{GHz}$. The degree of circular polarization expected from synchrotron radiation for such optical depths does not exhibit a peak, but instead passes through zero (e.g. Pacholczyk 1977).

Moreover, explanations based on synchrotron emission are not viable if the jet is composed mainly of $\mathrm{e}^{ \pm}$pairs (see e.g., Kaiser \& Hannikainen 2002). In this case little intrinsic circularly polarized emission is expected, as the circular polarization from each electron is equal in magnitude but of opposite sign to each positron.

\subsection{Circular polarization variations}

It is possible to explain the temporal variability of the circular polarization as a result of the emission from a succession of evolving ejections.

The circular polarization variability is qualitatively reproduced by a simple model in which the emission from the multiple ejections each evolves from optically thick to thin, and in which the circular polarization changes sign as it does so. The circular polarization observed is the sum of the contributions from all previous ejections.

Hannikainen et al. (2000) identify three separate ejection events in the period TJD 9579-9598 on the basis of the linearly polarized emission detected by ATCA at TJDs 9582, 9587 and 9591.

As the first burst commences it is initially optically thick and left-hand (negatively) circularly polarized. The circular polarization changes sign as the emission becomes optically thin, as observed in the data. However, at TJD 9582, a second burst occurs. If it, too, is initially negative circularly polarized, this explains the rapid decrease in the apparent level of circular polarization at this epoch. However, as this burst also becomes optically thin after $\sim 1$ day, the nett circular polarization returns to a high value, since the contributions from the two bursts are now both positive.

It is difficult to verify the model with data at later times because the high linear polarization causes large measurement uncertainties in the circular polarization due to instrumental polarization leakage. However, the negative circular polarization at TJD 9591 at $2.4 \mathrm{GHz}$ may be due to the emission from the third outburst.

This model has an obvious prediction. High resolution (VLBI) polarimetry capable of resolving individual ejecta at early epochs would detect circular polarization of order a few percent, and which reverses sign as the emission becomes optically thin to synchrotron radiation.

\subsection{Comparison with GRS $1915+105$}

Fender et al. (2002) report circular polarization associated with multiple outbursts in the Galactic superluminal source GRS 1915+105. The circular polarization evolves on time scales of $\sim 3$ hours and is strongest at the lowest measured frequency, $1.4 \mathrm{GHz}$, as observed in GRO J1655-40.

There is evidence for multiple components at varying optical depths; total flux density and spectral index lightcurves indicate there are at least four ejection events in the 2001 January outburst. The circular polarization is also reported to be coming from the parts of the system with "appreciable optical depth". The variability in the circular polarization could well be driven by opacity evolution in these components. This is consistent with what is observed in GRO J1655-40, where the circular polarization is also strongest at $\tau \sim 1$.

\subsection{Comparison with compact flat-spectrum AGN}

Circular polarization is detected in a number of AGN (e.g. Komesaroff et al. 1984; Raynder et al. 2000). In particular, it is found in the parsec-scale jets of blazars such as $3 \mathrm{C} 273$, 3C 279 and 3C 84 (Homan \& Wardle 1999; Homan et al. 2001). The circular polarization from these sources is highly variable. We also note that scintillation variability measurements imply degrees of circular polarization about $4 \%$ in some AGN (Macquart et al. 2000; Macquart 2002), comparable to what we observe in GRO J1655-40 (see Fig. 3).

There is evidence that the degree of circular polarization is stronger in flat-spectrum sources (Rayner et al. 2000, see also Conway et al. 1971; Roberts et al. 1975). There is, however, no correlation between the degree of circular and linear polarization.

The circularly polarized emission from flat-spectrum sources is mainly from the compact core region. The emission from their extended jets/ejecta is more linearly polarized (Homan \& Wardle 1999). Observations of the radio flaring in 3C 273 between 1994 and 1997 show that the linear polarization increases only after the peak of the flare and when total flux density had decreased substantially (Stevens et al. 1998).

In this sense, the polarization behaviour in GRO J1655-40 and 3C 273 are similar. In both cases the circular polarization originates from a compact region. Moreover, the linear polarization increases only in the later stage of the flare.

Wu et al. (2002) and Stevens et al. (2002) suggest that the temporal and spectral evolution of GRO J1655-40 and compact flat-spectrum radio sources can be explained in a unified framework based on the generalized-shock model of Marscher $\&$ Gear (1985). If the circularly polarized emission indeed originates from a compact region where the shocks are developed, the physical conditions in the environments near the compact object should play an important role for the emission and radiative transport processes responsible for the circular polarization. In particular, the intense radiation from the accretion disk could cool the relativistic electrons accelerated by the shocks, and hence determine the early stages of the shock evolution. This in turn would determine the source spectral evolution, as well as the polarization properties at the onset of the outburst. 
These issues will be discussed more quantitatively in Macquart $\& \mathrm{Wu}$ (in preparation).

\section{Conclusions}

We report the detection of circular polarization in the Galactic X-ray binary GRO J1655-40. The circular polarization changes sign at the lowest observed frequency, $1.4 \mathrm{GHz}$ during the commencement of the outburst. This coincides with the evolution of the spectrum from being inverted near this frequency to a pure power law. As such, it suggests that the circular polarization changes sign and peaks as the synchrotron opacity decreases to be comparable to unity. Such behaviour suggests that the circular polarization originates due to polarization conversion in the relativistic plasma associated with the source.

Acknowledgements. K.W. acknowledges the support from Australian Research Council through an ARC Australian Research Fellowship. DCH acknowledges the support of a PPARC postdoctoral research grant to the University of Southampton and financial support from the Academy of Finland. The Australia Telescope is funded by the Commonwealth Government of Australia for operation as a national facility by the CSIRO. The authors thank Rob Fender for comments on the paper.

\section{References}

Bower, G. C., Falcke, H., \& Backer, D. C. 1999, ApJ, 523, L29

Brunthaler, A., Bower, G. C., Falcke, H., \& Mellon, R. R. 2001, ApJ, $560, \mathrm{~L} 123$

Campbell-Wilson, D., \& Hunstead, R. W. 1994a, IAU Circ., 6052

Campbell-Wilson, D., \& Hunstead, R. W. 1994b, IAU Circ., 6055

Cheng, A. Y. S., Pacholczyk, A. G., \& Cook, K. H. 1985, ApJ, 297, 639

Conway, R. G., Gilbert, J. A., Raimond, E., \& Weiler, K. W. 1971, MNRAS, 152, $1 \mathrm{P}$

Fender, R., Rayner, D., Norris, R., Sault, R. J., \& Pooley, G. 2000, ApJ, 530, L29

Fender, R. P., Rayner, D., McCormick, D. G., et al. 2002, MNRAS, 336, 39

Frater, R. H., Brooks, J. W., \& Whiteoak, J. B. 1992, J. Elec. Electron. Eng. Aust., 12, 103

Hannikainen, D. C., Hunstead, R. W., Campbell-Wilson, D., et al. 2000, ApJ, 540, 521

Harmon, B. A., Wilson, C. A., Zhang, S. N., et al. 1995, Nature, 374, 703
Hjellming, R. M., \& Johnston, K. J. 1988, ApJ, 328, 600

Hjellming, R. M., \& Rupen, M. P. 1995, Nature, 375, 464

Homan, D. C., \& Wardle, J. F. C. 1999, AJ, 118, 1942

Homan, D. C., Attridge, J. M., \& Wardle, J. F. C. 2001, ApJ, 556, 113

Kaiser, C. R., \& Hannikainen, D. C. 2002, MNRAS, 330, 225

Komesaroff, M. M., Roberts, J. A., Milne, D. K., Rayner, R. T. \& Cooke, D. J. 1984, MNRAS, 208, 409

Legg, M. P. C., \& Westfold, K. C. 1968, ApJ, 154, 99

Macquart, J.-P. 2002, Publ. Astron. Soc. Aust., 19, 43

Macquart, J.-P., \& Melrose, D. B. 2000, ApJ, 545, 798

Macquart, J.-P., Kedziora-Chudczer, L., Rayner, D. P., \& Jauncey, D. L. 2000, ApJ, 538, 623

Marscher, A. P., \& Gear, W. K. 1985, ApJ, 298, 114

Melrose, D. B. 1971, Ap\&SS, 12, 172

Orosz, J. A., \& Bailyn, C. D. 1997, ApJ, 477, 876 (Erratum 482, 1086)

Pacholczyk, A. G. 1973, MNRAS, 163, 29P

Pacholczyk, A. G. 1977, Radio Galaxies (Permagon Press: Oxford)

Rayner, D. P. 2000, Ph. D. Thesis Circular polarization of active galaxies (University of Tasmania)

Rayner, D. P., Norris, R. P., \& Sault, R. J. 2000, MNRAS, 319, 484

Reynolds, J. E. 1994, ATNF Tech. Document AT/39.3/040, Australia Telescope National Facility

Roberts, J. A., Roger, R. S., Ribes, J. C., et al. 1975, Austr. J. Phys., 28,325

Ruszkowski, M., \& Begelman, M. C. 2002, ApJ, 573, 485

Sault, R. J., \& Macquart, J.-P. 1999, ApJ, 526, L85

Sault, R. J., Killeen, N. E. B., \& Kesteven, M. J. 1991, ATNF Tech. Document AT/39.3/015, Australia Telescope National Facility

Sault, R. J., Teuben, P. J., \& Wright, M. C. H. 1995, in Astronomical Data Analysis Software and Systems IV, ed. R. Shaw, H. E. Payne, \& J. J. E. Hayes, ASP Conf. Ser., 77, 433

Shahbaz, T., van der Hooft, F., Casares, J., Charles, P. A., \& van Paradijs, J. 1999, MNRAS, 306, 89

Soria, R., Wickramasinghe, D. T., Hunstead, R. W., \& Wu, K. 1998, ApJ, 495, L95

Stevens, J. A., Hannikainen, D. C., Wu, K., Hunstead, R. W., \& McKay, D. 2002, MNRAS, submitted

Stevens, J. A., Robson, E. I., Gear, W. K., et al. 1998, ApJ, 502, 182

Tingay, S. J., Jauncey, D. L., Preston, R. A., et al. 1995, Nature, 374, 141

Wardle, J. F. C., \& Homan, D. C. 2001, in Particles and Fields in Radio Galaxies, ed. R. A. Laing, \& K. M. Blundell, ASP Conf. Proc., 250

Wardle, J. F. C., Homan, D. C., Ojha, R., \& Roberts, D. H. 1998, Nature, 395, 457

Wu, K., Stevens, J. A., \& Hannikainen, D. C. 2002, Publ. Astron. Soc. Aust., 19, 91

Zhang, S. N., Wilson, C. A., Harmon, B. A., et al. 1994, IAU Circ., 6046 